\title{
DISINHIBITION TRANSMITS FROM TELEVISION TO YOUNG CHILDREN
}

\author{
Yusuke MORIGUCHI ${ }^{1)}$, Wakako SANEFUJI ${ }^{2)}$ and Shoji ITAKURA ${ }^{1)}$ \\ ${ }^{1)}$ Kyoto University, Japan, ${ }^{2)}$ Kyushu University, Japan
}

\begin{abstract}
The present study examined whether young children's behaviors in the Dimensional Change Card Sorting task (DCCS task) could be influenced by their observation of another person performing the task on video. In Experiment 1,3-, 4-, and 5-year-old children observed that an actor on video was sorting cards according to one rule (e.g., shape rule). Then they were asked to sort the cards according to the other rule (e.g., color rule). Result showed that 3-year-olds failed to use the second rule whereas 4- and 5-year-olds used the second rule. Experiments 2 further investigated whether children's performance following the televised demonstration was different from their performance following the live demonstration of the same actions. Results showed that 3-year-old children failed to use the second rule in spite of the presentation modality (live or video). We conclude that disinhibition can transmit from television to children as it transmits from one person to children.
\end{abstract}

Key words: inhibitory control, perseverative errors, understanding Television, preschool children

Imagine a situation your 3-year-old daughter is putting her toys into a box while you are watching TV next to your daughter. On a TV program, an actor makes an attempt to eat some toys nearby. When you are looking aside from your daughter, she may be attracted to the actor on the TV program and mimic the actor's actions. Even when you look back at your daughter and asked her not to mimic the actions, your daughter may not stop and perseverate to the actions. The present study aimed to address these kinds of issue in the light of the development of inhibitory control.

Inhibitory control refers to the ability to inhibit irrelevant and inappropriate actions, for the attainment of a specific goal. This ability plays an important role in multiple areas of child development such as intelligence and social interaction (e.g., Blair, 2002; Carlson \& Moses, 2001; Dempster,1992; Diamond, Kirkham, \& Amso, 2002; Harnishfeger \& Bjorklund, 1993; Kochanska, Murray, Jacques, Koenig, and Vandegeest, 1996). Extensive research to date suggests that between three and five years of age, children's inhibitory control undergoes rapid development (Carlson, 2005).

Yusuke Moriguchi and Shoji Itakura, Department of Psychology, Graduate School of Letters, Kyoto University. Wakako Sanefuji, Department of Psychology, Graduate School of Human-Environment Studies, Kyushu University.

The research reported here was supported by a grant from JSPS (No: 13610087, 16500161) to Shoji Itakura, and by the 21 st Century COE Program (D-2 to Kyoto University), MEXT, Japan. The authors thank Chiyo Ito for help with data collection.

Correspondence concerning this article should be addressed to Yusuke Moriguchi, Department of Psychology, Graduate School of Letters, Kyoto University, Yoshida-honmachi, Sakyo-ku, Kyoto 606-8501 Japan (e-mail: m.yusuke@bun.kyoto-u.ac.jp). 
One task that has been used to measure children's inhibitory control ability is the dimensional change card sorting task (DCCS: Zelazo, Frye, \& Rapus, 1996). In this task, children are asked to sort cards that have two dimensions (e.g., color and shape: red boats, red rabbits, blue boats, and blue rabbits). There are two phases in this task. In the first phase, children are asked to sort cards according to one dimension (e.g., color), for several trials. In the second phase, the experimenter tells children that the sorting rule is to be changed and asks them to sort the cards according to the other dimension (e.g., shape). The results have shown that older preschoolers can switch easily from one dimension to the other in their sorting behavior. In contrast, younger preschoolers have difficulty, after correctly sorting the cards according to the first dimension in the first phase, in switching their sorting according to the second dimension in the second phase. Instead, they perseverate and continue to sort the cards according to the first dimension.

Recent investigations have shown that inhibitory control could be affected by social factors. Many studies suggested that typically and atypically developing children have shown robust correlation between inhibitory control and social cognition, such as theory of mind (e.g., Carlson, Moses, \& Breton, 2002; Hughes, Dunn, \& White, 1998; Perner, Lang, \& Kloo, 2002). Most of the researchers proposed that inhibitory control ability is necessary for developing theory of mind (Carlson, Mandell, \& Williams, 2004; Flynn, O’Malley, \& Wood, 2004; Moses, 2005; Sabbagh, Xu, Carlson, Moses, \& Lee, 2006) while Perner (1998) suggested that theory of mind understanding may contribute to improvement in children's inhibitory control ability.

Another line of evidence comes from studies which showed that action observation may elicit perseverative tendencies. Longo and Bertenthal (2006) showed that young infants committed perseverative errors after the observation of another person's actions. In the Piagetian A-not-B task, infants searched a toy hidden at location A or observed an experimenter search the toy. After the toy was hidden at location B, infants in both conditions perseverated in reaching to A. Theorists proposed that the perseverative errors observed in 9-month-olds may be similar to 3-year-old children's perseveration (such as in DCCS task) because both perseverations might be strongly correlated to the function of prefrontal cortex (Diamond, 2002). However, it appears that two perseverative errors were clearly different in terms of task complexity and task demand. More recently, Moriguchi, Lee and Itakura (2007) showed that social observation could affect children's tendencies to commit perseverative errors in a modified DCCS task. In the task, instead of sorting cards themselves, preschoolers watched an adult model sorting the cards according to one rule (e.g., the shape rule), after which they were asked to sort according to a different rule (e.g., the color rule).Results showed that most 3-year-olds failed to use the second rule and perseverated to the first, observed rule. On the other hand, 4-and 5-yearolds successfully used the second rule. From these results, Moriguchi et al. suggested that social observation can lead to disinhibition in a manner similar to the typical findings in the DCCS task that young children fail to inhibit a first rule to sort cards and keep using the rule even when they are asked to use the second rule.

It appears that children's inhibitory control could be affected by their observation of another person's actions. One question of particular interest here is whether young 
children's inhibitory control would be affected by TV or video. Children's social observation in everyday life also includes observation of actors' behaviors on TV or video. Thus it could be that the same influence is induced even when young children observe another person's behaviors on TV or video. Indeed, it is well known that TV and video plays an important role in children's everyday life. Many young children have extensive experience with TV or video (Huston, Wright, Marquis, \& Green, 1999; Jordan \& Woodward, 2001) and they learn vocabulary and concepts from television (Hughes et al., 1999; Rice, Huston, Truglio, \& Wright 1990; Truglio, Scheiner, Segui, \& Chen, 1999; Wright \& Huston, 1995; Wright et al., 2001). Also, experimental studies suggested that children develop rapidly understanding television until 3-year-old. Most 2.5-year-old children can use information included on TV to guide their actual actions (Troseth \& DeLoache, 1998). Three-year-olds could imitate actions presented by a televised demonstration as well as by a live demonstration whereas 2- and 2.5-year-olds performance following the televised demonstration is inferior to their performance following the live demonstration of the same actions (Hayne, Herbert, \& Simcock, 2003; McCall, Parke, \& Kavanaugh, 1977).

Given these findings, not only observation of people live but observation of people on TV or video would have the impact on children's inhibitory control. In the current study, we examined whether young children would commit perseverative errors merely after watching televised demonstration of the DCCS task. To test this, we adapted the paradigm used in Moriguchi et al. (2007). Preschoolers watched an adult model sorting cards according to one rule (e.g., the shape rule) on video, after which they were asked to sort cards according to a different rule (e.g., the color rule).

As participants, 3-, 4- and 5-year-old children were chosen because previous researchers (e.g., Zelazo \& Reznik, 1991) has shown that only by 3-year-old are children ready for these kinds of tasks. In Experiment 1, we examined whether preschoolers would show perseverative errors and fail to sort cards according to the second rule after merely watching an adult model sorting cards according to the first rule on video. Experiments 2 further investigated whether children's performance following a televised demonstration was different from their performance following a live demonstration of the same actions.

\section{EXPERIMENT 1}

\section{METHOD}

\section{Participants}

Twenty 3-year-old children $(M=43.1$ months, range $=36$ months to 47 months, 12 boys and 8 girls $)$, twenty 4-year-old children ( $M=54.1$ months, range 49 months to 60 months, 11 boys and 9 girls $)$ and twenty 5-year-old children ( $M=66.7$ months, range 61 months to 72 months, 13 boys and 7 girls) were recruited from nursery schools in Fukuoka as participants. Most participants came from a middle-class back-ground.

\section{Materials}

Laminated cards $(3.5 \mathrm{~cm} \times 7.0 \mathrm{~cm})$ were used as stimuli. For the warm-up phase, there were a red star card, a blue cup card, a blue star card, and a red cup card. For the observation and sorting phase, there were 
two trays $(4.5 \mathrm{~cm} \times 0.5 \mathrm{~cm} \times 15.0 \mathrm{~cm})$, one of which contained a card depicting a red star and the other contained a card depicting a blue cup. There were sorting cards depicting either a blue star or a red cup (three for each). A video of the demonstration was presented by a Dell note-book sized personal computer.

\section{Procedure}

Each child was tested individually for about 5 minutes. A child was seated at a table next to an experimenter. There were three phases in this task: warm-up phase, observation phase, and sorting phase. On warm-up phase, the child was presented with four cards (a red star card, a blue cup card, a blue star card, and a red cup card). The child was asked to name all the shapes and their colors. Then the same cards were presented on the computer screen and the child was asked to match cards in front of him/her with the cards presented on the computer screen. This was to ascertain whether the child could identify the cards on the computer screen with the cards in front of him/her. If the child failed on this phase, the child was asked to match the cards again. If the child failed again, the child was excluded from the analyses. After that, the experimenter introduced two trays and sorting cards, and announced the general rule of the task ("There are two ways to sort the cards, color and shape. In this game, I will tell you whether you should sort the cards according to its color or its shape.")

During observation phase, the child was asked to watch a video. On video, there were a model and two trays which were the same as those for children. Then he/she was told that the model would put sorting cards into the trays ("Now she [the model] is going to sort the cards first. Please watch carefully"). The model sorted the cards according to one dimension. Half of the children saw the model sorting the cards according to the shape dimension and the other half saw the model sorting according to the color dimension. During the observation, the child was not given an explicit rule. Instead, he/she was encouraged to watch the video. The model performed four trials (two trials for a blue star and two trials for a red cup).

During sorting phase, the experimenter introduced to the child the trays and sorting cards again. The child was instructed to have a game ("Now, it is your turn. We are going to play a game. This game is different from the game she [the model] played.") The child was instructed to sort the cards according to the second rule. For example, when the model sorted the cards according to the shape dimension, children were asked to sort the cards according to the color dimension ("Your game is a color game. The color game is different from the shape game. In the color game, all the red ones go here and all the blue ones go there.") The child was given six sorting trials. On every trial, the experimenter told the child the rule of the game, randomly selected a sorting card for them to sort ("Where does this card go in the color game?") The child was required to place the card in one of the two trays. They were not given any feedback about whether they sorted the cards correctly.

\section{RESULT AND DISCUSSION}

One 3-year-old child was excluded from the analysis because she failed to answer the questions correctly on the warm-up phase. The rest of the children answered the questions correctly. Children were scored as "correct" if they sorted a card correctly according to the dimension instructed by the experimenter. Preliminary analyses using chi-square tests showed no significant differences in children's scores in the shape and color games, and therefore the data for the two types of games were combined for the subsequent analyses.

We examined whether data at each age group were different from what would be expected by chance. Expected frequencies were based on a chance distribution of 1/64, 6/ $64,15 / 64,20 / 64,15 / 64,6 / 64,1 / 64$ for participants who made $0,1,2,3,4,5,6$ correct responses, respectively. We classified children according to whether children made $0-1$ correct responses, 2-4 correct responses, and 5-6 correct responses (see Fig. 1). As a result, the expected frequencies were 7/64, 50/64 and 7/64, respectively. Chi-square tests revealed that distributions of scores of 3- , 4- and 5-year-old children were all significantly 


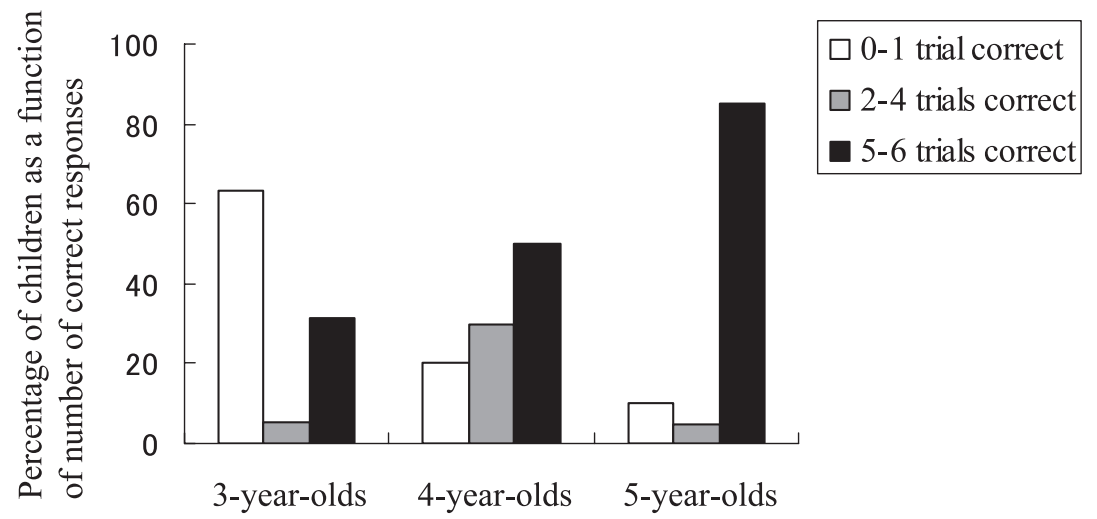

Fig. 1. Correct response distributions of children in each age group in Experiment 1.

different from chance: $\chi^{2}(2, N=38)=20.882, p<.001, \chi^{2}(2, N=40)=9.776, p<.01$, and $\chi^{2}(2, N=40)=24.258, p<.001$, respectively. The results suggested that most of the children at each age group were classified into 0-1 trial correct responses (perseverative errors) or 5-6 trials correct (passing the task).

Next we examined whether there was age-related change in children's performance distribution (i.e., 0-1 trial correct, 2-4 trials correct, 5-6 trials correct). Most of the 3-yearold children committed perserverative errors whereas few 4- and 5-year-old children show the perserverative tendencies. The chi-square test revealed that younger children were more likely to commit perserverative errors than older children $\chi^{2}(4, N=59)=21.313$, $p<.001)$. Further chi-square tests showed significant differences between three- and four-year-olds $\chi^{2}(1, N=39)=8.551, p<.02$, three- and five-year-olds $\chi^{2}(1, N=39)=$ $12.386, p<.003$, and between four- and five-year-olds, $\chi^{2}(1, N=40)=6.052, p<.05$

In Experiment1, more than half of the 3-year-old children failed to follow the second rule on at least five trials even when the experimenter asked them to use the rule. Instead, they followed the rule presented on video and perseverated to the rule. The current results confirmed the finding that 3-year-olds's inhibitory control was affected by the observation of another person's actions (Moriguchi et al., 2007). Furthermore, the results of Experiment1 suggested that young children's inhibitory control was influenced by observation of an actor's actions on TV or video. In other words, social observation, not only observation of another person's behaviors live but observation of another person's behaviors on TV or video, can lead young children to disinhibition.

On the other hand, only $20 \%$ of the 4 -year-olds and $10 \%$ of the 5 -year-olds committed perseverative errors. Most of the older children scored correctly on at least five trials. This developmental change was consistent with the earlier studies which suggested that children improve inhibitory control ability during preschool years (Carlson, 2005; Zelazo et al., 1996). 


\section{EXPERIMENT 2}

Experiment 1 suggested that children's behaviors in the DCCS task could be affected by observing the televised demonstration of the same task. In Experiment 2, we compared directly the impact of a televised demonstration on young children with that of a live demonstration. We predicted that the impact of a televised demonstration on children's inhibitory control might be the same as that of a live demonstration. This prediction was based on a suggestion that children could extract information from a demonstrator on TV as well as a live demonstrator until the age of 36 months (Hayne et al., 2003).

As participants, we chose 3-year-old children because Experiment 1 showed that few 4-and 5-year-old children committed perseverative errors. Children were given two cardsorting tasks: in one task, children observed the televised demonstration and in the other task children observed the live demonstration.

\section{METHOD}

\section{Participants}

Twenty one 3 -year-old children $(M=43.6$ months, range $=38$ months to 50 months, 12 boys and 9 girls) were recruited from nursery schools in Fukuoka as participants. Most participants came from a middleclass back-ground. None of the participants in Experiment 1 took part in Experiment 2.

\section{Materials}

The materials used in video condition were the same as in Experiment 1. For live condition, there were two trays $(4.5 \mathrm{~cm} \times 10.5 \mathrm{~cm} \times 15.0 \mathrm{~cm})$, one of which contained a card depicting a green car and the other contained a yellow house. There were sorting cards depicting either a yellow car or a green house (six for each)

Procedure

Each child participated both in video condition and in live condition. Each child was tested individually for about 10-15 minutes. In the video condition, there were a child, an experimenter and a computer. In the live condition, there were a child, an experimenter and a model. The order of conditions (the video condition vs the live condition first) was counterbalanced.

Video condition The video condition was identical to Experiment 1.

Live condition The child was seated at a table. The experimenter sat at a table across from the child, and the model sat in front of the child. There were three phases: warm-up phase, observation phase, and sorting phase. On the warm-up phase, the child was presented with trays and sorting cards and asked to name all the shapes (house, car) and their colors (green, yellow). After labeling, the experimenter announced the general rule of the task.

On the observation phase, the child was told that the model would sort cards first. The live model sorted cards according to one dimension (e.g., shape). All other aspects of the live demonstration were matched to the televised demonstration including the speed at which the actions were performed. After sorting cards, the model made an excuse and left the room.

The sorting phase was identical to Experiment 1.

\section{RESULT AND DisCuSSION}

Two children were excluded from the analysis. They failed to answer the questions on the warm-up phase in both of the video and live condition. The rest of the children 


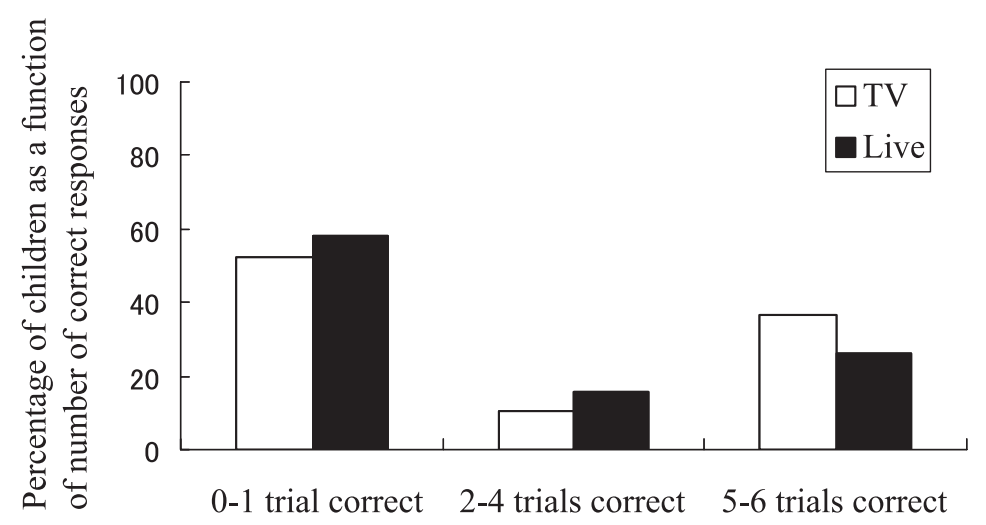

Fig. 2. Correct response distributions of 3-year-old children in each condition in Experiment 2.

answered the questions correctly. Preliminary analyses using a chi-square test showed no significant differences in children's scores in the shape and color games. Also, the task order did not affect children's performances. Therefore the data for these factors were combined for the subsequent analyses.

We examined whether data at each age group were different from what would be expected by chance as in Experiment 1. Chi-square tests revealed that distributions of scores of children in both conditions were significantly different from chance: live condition, $\chi^{2}(2, N=38)=15.054, p<.001$, and video condition, $\chi^{2}(2, N=38)=17.549$, $p<.001$, respectively (Fig. 2). We conducted sign test to examine whether there was difference of performance distribution between conditions, and found that there were no significant differences between conditions, $p>.72$. Three children showed the better performances in the live condition than in the video condition while five children showed the opposite pattern. The rest of the children performed equally both in the live condition and the video condition. Next, the correlation of the performance distribution between conditions was examined using Spearman correlation, and we found a significant correlation between them, $r=.77 p<.001$.

The result of Expeperiment 1 was replicated. More than half of the 3-year-old children failed to use the second rule after watching another person's rule-use on video. Furthermore, children's performances after watching the televised demonstration were almost the same as their performances after watching the demonstration live. This result suggested that the impact of televised demonstration on children's inhibitory control was almost the same as that of live demonstration.

\section{GENERAL DisCuSSION}

The present study investigated whether young children's behaviors in the DCCS task could be influenced by their observation of another person doing the same task on video. 
In Experiment 1, most of the 3-year-old children failed to sort cards after watching a televised demonstration. They followed a rule presented on video and continued to use the rule on most of the trials even when they were asked to use the second rule. On the other hand, most of the 4- and 5-year-olds were able to use the second rule when they were instructed to use the rule. In Experiment 2, we showed that 3-year-olds' performance following a televised demonstration is as low as their performance following a live demonstration. The children showed perseverative errors after watching the televised demonstration as well as after watching the live demonstration.

Our results are consistent with the recent investigations that the social factors might have impact on young children's inhibitory control. Towse, Redbond, Houston-Price \& Cook (2000) found that, in the DCCS task, an experimenter's demonstration of sorting according to the second rule in the second phase reduced children's tendency to perseverate. Moriguchi et al. (2007) reported that observation of another person's actions influenced 3-year-olds' inhibitory control and led them to perseverative errors. The present research extends the earlier findings in an important way by showing that the impact of social factor is not limited to the observation of another person's actions live. Young children's inhibitory control was also affected by their observation of an actor on TV or video. When children observe another person's actions through a TV screen, observation of the actions have a contagious effect on young children. In addition, the impact of the actor on video was almost the same as that of the model live. These suggested that disinhibition can transmit from television to children as it transmits from one person to children.

Although the present study did not address whether the developmental pattern in the video condition was the same as in the live condition, we believe that two developmental pattern would be identical. Anderson and Pempek (2005) proposed the concept of "video deficit", with which young children learn less from video than from real-life experiences. According to the recent evidence, the video deficit might disappear until the age of 3 (Anderson \& Pempek, 2005; McCall et al., 1977; Troseth, 2003; Troseth, Saylor, \& Archer, 2006) despite the fact that even older children fail to discriminate video world from real world under some circumstances (e.g., Kimura \& Kato, 2006). In addition, our previous study (Moriguchi et al., 2007) suggested that 4-and 5-year-old children's actions were not affected by a live model's actions, which was replicated by the present study using a video model (Experiment 1).Given the evidence, the developmental trajectory in video condition appears to be identical to that in live condition.

One might argue that children tended to use the observed rule after watching video because they liked to follow an adult actor's actions rather than to follow an experimenter's verbal instruction. However, in a previous study, Moriguchi et al. (2007) showed that children did not follow blindly another person's actions in the live demonstration version of the task. Most of the 3-year-olds made perseverative errors when the model sorted the cards normally (as in the present study) or when the adult model was confident about her sorting. On the other hand, when the adult model appeared uncertain whether she sorted the cards correctly, most children never committed perseverative errors. This might be the case with the televised demonstration version of 
the task because the present result showed that children's performance following a televised demonstration was strongly correlated with the performance following a demonstration live.

Video stimulus is important for our argument regarding the impact of social factors on children's inhibitory control for two reasons. One reason is, as described above, that children have extensive experience with video or TV (Huston, et al.,1999: Jordan \& Woodward, 2001) and how actors on video or TV affect children's actions is itself worth investigating. The other reason is that in contrast to live models, children can not interact and communicate with video models. In case of a live model, children can communicate directly with the model, which could affect young children's performances in the previous study (live condition). However, the current results showed that young children's actions were strongly influenced by the video model as well as the live model. The results suggested that the interaction between children and the model was not crucial for the task performances. Given the results, we suggest that observation of human's actions itself rather than interaction or communication between models and children could cause young children's disinhibition.

The present results confirmed that children develop inhibitory control ability during preschool years. In the standard DCCS task, many 3-year-old children fail to switch rules after they sort the cards according to the first rule (Zelazo et al., 1996). On the other hand, most 4- and 5-year-old children can switch rules easily. In the current study, although children did not sort cards according to the first rule, the same developmental change was observed: younger children were more likely to fail to sort the cards according to the second rule than older children.

One remaining question is the reason why social factors have significant impacts on children's inhibitory control. There are two possible interpretations. One is that another person's actions simply captured children's attention and directed it to the dimension (e.g., color) the person used. Once children's attention focused the dimension, they might have had the difficulty with switching their attention from the dimension to the other dimension. The other possibility is that children's tendencies to imitate another person's actions led them to the preseverative errors. After observing another person's sorting, 3year-old children might have reproduced the same actions. There is evidence that 2- to 4year-old children show the tendencies to faithfully reproduce another person's solutions in a tool-using task even when the solutions are inefficient and a more efficient solution is available (Nagell, Olguin, \& Tomasello, 1993; Horner \& Whiten, 2005). At this point, we can not exclude these two possibilities. Therefore further studies are needed to test the validity of the explanations.

Nevertheless, the fact that social factors may affect children's inhibitory control in some situations (e.g., when watching TV) might lead to our understanding how social experience can exert an influence on the development of inhibitory control ability. There is some evidence that specific interventions could improve children's inhibitory control ability. For example, Kloo and Pener (2003) showed that an intensive and explicit training improved markedly children's performance on the DCCS task. Rueda, Rothbart, McCandliss, Saccomanno, and Posner (2005) suggested that an educational intervention 
might exert an influence on both behavioral scores of executive attention measured by the attention network test and the event-related potentials data during attention network test performance. Along with the studies, developing our paradigm might lead to the findings that social observation is important for the development of inhibitory control.

\section{REFERENCE}

Anderson, D. R., \& Pempek, T. A. 2005. Television and very young children. American Behavioral Scientist, 48, 505-522.

Blair, C. 2002. School readiness: Integrating cognition and emotion in a neurobiological conceptualization of children's functioning at school entry. American Psychologist, 57, 111-127.

Carlson, S. M. 2005. Developmentally sensitive measures of executivefunction in preschool children. Developmental Neuropsychology, 28, 595-616.

Carlson, S. M., Mandell, D. J., \& Williams L. 2004. Executive function and theory of mind: Stability and prediction from ages 2 to 3. Developmental Psychology, 40, 1105-1122.

Carlson, S. M., \& Moses, L. J. 2001. Individual differences in inhibitory control and children's theory of mind. Child Development, 72, 1032-1053.

Carlson, S. M., Moses, L. J., \& Breton, C. 2002. How specific is the relation between executive function and theory of mind? Contributions of inhibitory control and working memory. Infant and Child Development, 11, 73-92.

Dempster, F. N. 1992. The rise and fall of the inhibitory mechanism: Toward a unified theory of cognitive development and aging. Developmental Review, 12, 45-75.

Diamond, A. 2002. Normal development of prefrontal cortex from birth to young adulthood: Cognitive functions, anatomy, and biochemistry. In D. T. Stuss \& R. T. Knightl (Ed) Principles of frontal lobe function (pp 466-503). Oxford, England: University Press

Diamond, A., Kirkham, N., \& Amso, D. 2002. Conditions under which young children can hold two rules in mind and inhibit a prepotent response. Developmental Psychology, 38, 352-362.

Flynn E., O’Malley C., \& Wood D.2004. A longitudinal, microgenetic study of the emergence of the false belief understanding and inhibition skills. Developmental Science, 7, 103-115.

Harnishfeger, K. K., \& Bjorklund, D. F. 1993. The ontogeny of inhibition mechanisms: Arenewed approach to cognitive development. In M. L. Howe \& R. Pasnak (Eds.), Emerging themes in cognitive development. Vol. I: Foundations (pp. 28-49). NewYork: Springer-Verlag.

Hayne, H., Herbert, J., \& Simcock, G. 2003. Imitation from television by 24- and 30-month-olds. Developmental Science, 6, 254-261.

Horner, V., \& Whiten, A. 2005. Causal knowledge and imitation/emulation switching in chimpanzees (Pan troglodytes) and children (Homo sapiens). Animal Cognition, 8, 164-181.

Hughes C., Dunn, J., \& White, A. 1998. Trick or treat?: Uneven understanding of mind and emotion and executive dysfunction in "hard to manage" preschoolers. Journal of Child Psychology and Psychiatry, 39, 981-994.

Hughes, K., Peyton, V., Hruda, L., O’Brien, M., Huston, A., \& Caldera, Y. 1999. Family characteristics and child outcomes associated with toddlers' television viewing. Poster session presented at the biennial meeting of the Society for Research in Child Development, Albuquerque, NM.

Huston, A. C., Wright, J. C., Marquis, J., \& Green, S. B. 1999. How young children spend their time: Television and other activities. Developmental Psychology, 35, 912-925.

Jordan, A.B., \& Woodward, E.H., IV. 2001. Electronic childhood: The availability and use of household media by 2- to 3-year-olds. Zero to Three, 22, 4-9.

Kimura, M., \& Kato, Y. 2006. youjino video eizou rikaino hattatsu ("Young children's understanding of video images"). Japanese Journal of Developmental Psychology, 17, 126-137. (in Japanese with English abstracts).

Kochanska, G., Murray, K. T., Jacques, T. Y., Koenig, A. L., \& Vandegeest, K. A. 1996. Inhibitory control in young children and its role in emerging internalization. Child Development, 67, 490-507. 
Kloo, D., \& Perner, J. 2003. Training transfer between card sorting and false belief understanding: Helping children apply conflicting descriptions. Child Development, 74, 1823-1839.

Longo, M. R., \& Bertenthal, B. I. 2006. Common Coding of Observation and Execution of Action in 9Month-Old Infants, Infancy, 10, 43-59.

McCall, R. B., Parke, R. D., \& Kavanaugh, R. D. 1977. Imitation of live and televised models by children one to three years of age. Monographs of the Society for Research in Child Development, 42 (5, Serial No. 173).

Moriguchi, Y., Lee, K., \& Itakura, S. 2007. Social transmission of disinhibition in young children. Developmental Science, 10, 481-491.

Moses, L. 2005. Executive function and children's theory of mind. In B. F. Malle \& S D. Hodges (Eds.), Other minds: How humans bridge the divide between self and others, (pp. 11-25). New York: Guilford Press.

Nagell, K, Olguin, R. S., \& Tomasello, M. 1993. Processes of social learning in the tool use of chimpanzees and human children. Journal of Comparative Psychology, 107, 174-186.

Perner, J. 1998. The meta-intentional nature of executive function and theory of mind. In P. Carruthers \& J. Boucher (Eds), Language and thought (pp. 270-283) Cambridege, UK: Cabbridege University Press.

Perner, J., Lang, B., \& Kloo, D. 2002. Theory of mind and self-control: More than a common problem of inhibition. Child Development, 73, 752-767.

Rice, M. L., Huston, A.C., Truglio, R., \& Wright, J. 1990. Words from "Sesame Street”: Learning vocabulary while viewing. Developmental Psychology, 26, 421-428.

Rueda, M. R., Rothbart, M. K., McCandliss, B. D., Saccomanno, L., \& Posner, M. I. 2005. Training, maturation, and genetic influences on the development of executive attention. Proceedings of the National Academy of Sciences, 102, 14931-14936

Sabbagh, M., Xu, F., Carlson, S. Moses, L., \& Lee, K. 2006. The development of executive functioning and theory-of-mind: A comparison of Chinese and U.S. preschoolers. Psychological Science, 17, 74-81.

Troseth, G. L. 2003. TV guide: Two-year-old children learn to use video as a source of information. Developmental Psychology, 39, 140-150.

Troseth, G. L., Saylor, M. M., \& Archer, A. H. 2006. Young children's use of video as a source of socially relevant information. Child Development, 77, 786-799.

Troseth, G. L., \& DeLoache, J. S. 1998. The medium can obscure the message: Young children's understanding of video. Child Development, 69, 950-965.

Towse, J. N., Redbond, J., Houston-Price, C. M. T., \& Cook, S. 2000. Understanding the dimensional change card sort. Perspectives from task success and failure. Cognitive Development, 15, 347-365.

Truglio, R. T., Scheiner, S., Segui, I., \& Chen, L. 1999. Sesame Street's science of discovery. Poster session presented at the biennial meeting of the Society for Research in Child Development, Albuquerque, NM.

Wright, J. C., \& Huston, A. C. 1995. Effects of educational TV viewing of lower income preschoolers on academic skills, school readiness, and school adjustment one to three years later: A report to Children's Television Workshop. Lawrence: Center for Research on the Influences of Television on Children, University of Kansas.

Wright, J. C., Huston, A. C., Murphy, K. C., St. Peters, M., Pinon, M., Scantlin, R., et al. 2001. The relations of early television viewing to school readiness and vocabulary of children from low-income families: The Early Windows Project. Child Development, 72, 1347-1366.

Zelazo, P. D., Frye, D., \& Rapus, T. 1996. An age-related dissociation between knowing rules and using them. Cognitive Development, 11, 37-63.

Zelazo, P. D., \& Reznick, J. S. 1991. Age-related asynchrony of knowledge and action. Child Development, 62, 719-735. 